\title{
ON THE JOIN OF SUBNORMAL SUBGROUPS
}

\section{A. J. VAN WERKHOOVEN}

ABSTRACT. Let $\boldsymbol{G}$ be the class of finitely generated groups. If the join of finitely many subnormal $\mathfrak{X}=s n \mathfrak{X}$ subgroups is always an $\mathfrak{X}$-group and $\mathfrak{Y}=\left\{s n, q, n_{0}\right\} \mathfrak{Y} \subseteq \mathfrak{W}$, then the join of finitely many subnormal $\mathfrak{X Y}$-subgroups is an $\mathfrak{X Y}$-group. If the subnormal coalition class $\mathfrak{X}$ and the class $\mathfrak{Y}=\left\{s n, q, n_{0}\right\} \mathfrak{Y}$ are such that whenever $A \in \mathfrak{X}, A$ has a maximum subnormal $\mathfrak{X}$-subgroup, then $\mathfrak{X}(\mathfrak{Y} \wedge(\mathfrak{b})$ is a subnormal coalition class $(\mathfrak{Y} \wedge \mathfrak{F}$ is the class of finitely generated Y-groups).

1. Introduction and notation. In this section we state our results. The notation used is discussed in 1.3 and 1.4.

1.1. Definition. The class $\mathfrak{X}$ is a subnormal coalition class if, whenever $H$ and $K$ are subnormal $\mathfrak{X}$-subgroups of $G$, their join $\langle H, K\rangle$ is a subnormal $\mathfrak{X}$-subgroup of $G$.

We establish a condition which implies that the class $\mathfrak{X}_{1} \mathfrak{X}_{2}$ is a subnormal coalition class, given that $\mathfrak{X}_{1}$ and $\mathfrak{X}_{2}$ are subnormal coalition classes.

THEOREM A. If the subnormal coalition class $\mathfrak{X}$ and the class $\mathfrak{Y}=$ $\left\{s n, q, n_{0}\right\} \mathfrak{Y}$ are such that whenever $A \in \mathfrak{X} \mathfrak{Y}, A$ has a maximum subnormal $\mathfrak{X}$-subgroup, then $\mathfrak{X}(\mathfrak{Y} \wedge(\mathfrak{b})$ is a subnormal coalition class. $\hat{\mathfrak{M}}_{\text {s. }}$.

We may take, for example, for the class $\mathfrak{Y}$ of Theorem $A$ the class

It is a consequence of 2.8 that whenever $\mathfrak{X}$ is a subnormal coalition class and $\mathfrak{Y}=\left\{s n, q, n_{0}\right\} \mathfrak{Y} \subseteq \mathfrak{G}$, an $\mathfrak{X} \mathfrak{Y}$-group has a maximum subnormal $\mathfrak{X}$-subgroup. Hence, as a corollary to Theorem $A$ we have

THEOREM B. If $\mathfrak{X}$ is a subnormal coalition class and $\mathfrak{Y}=\left\{s n, q, n_{0}\right\} \mathfrak{Y} \subseteq(\mathfrak{H}$ is a class of groups, then $\mathfrak{X Y}$ is a subnormal coalition class.

In Theorem $B$ we may take for the class $\mathfrak{Y}$ the classes $\mathfrak{F}, \hat{\mathfrak{P}}$, and $\mathfrak{G}^{s n}$. 1.2. Definition. If $\mathfrak{X}$ is a class of groups, then $G \in s_{0} \mathfrak{X}$ if and only if $G$ is the join of finitely many subnormal $\mathfrak{X}$-subgroups.

Presented to the Society, January 15, 1973; received by the editors December 21, 1972 and, in revised form, April 12, 1973.

AMS (MOS) subject classifications (1970). Primary 20F30, 20 E20.

Key words and phrases. Subnormal, subnormal coalition class. 
It is clear that every subnormal coalition class is $s_{0}$-closed. It is shown in [4] that the class of solvable groups is $s_{0}$-closed.

We also investigate conditions which imply that $\mathfrak{X}_{1} \mathfrak{X}_{2}$ is $s_{0}$-closed, given that $\mathfrak{X}_{1}$ and $\mathfrak{X}_{2}$ are $s_{0}$-closed classes.

TheOREM C. If $\mathfrak{X}=\left\{s n, s_{0}\right\} \mathfrak{X}$ and $\mathfrak{Y}=\left\{s n, q, n_{0}\right\} \mathfrak{Y} \subseteq \mathfrak{G}$ then $\mathfrak{X Y}=s_{0} \mathfrak{X}$.

We leave as an open question whether the condition “ $\mathfrak{X}=s n \mathfrak{X}$ " may be deleted from the hypothesis of Theorem C. A result in this direction is

THEOREM D. If $\mathfrak{X}=s_{0} \mathfrak{X}$, then $\mathfrak{X} \mathfrak{F}=s_{0} \mathfrak{X} \mathfrak{F}$.

The author is grateful to the referee for his helpful comments and suggestions.

1.3. The identity element and the group of order one are denoted by 1 . If $H$ is a subgroup of $G$, we write $H \subseteq G$ and denote by $|G: H|$ the index of $H$ in $G$. If $|G: H|=n$ and $G=\bigcup_{i=1}^{n} H a(i)$, we say that the set $T=\{a(1)$, $a(2), \cdots, a(n)\}$ is a right transversal for $H$ in $G$. If $H$ is a subnormal (normal) subgroup of $G$, we write $H \triangleleft \triangleleft G(H \triangleleft G)$ and denote by $s(G, H)$ the subnormal index for $H$ in $G$. If $G$ is generated by the subsets $T_{\alpha}$, $\alpha \in A$, we write $G=\left\langle T_{\alpha} \mid \alpha \in A\right\rangle$. $H^{g}$ denotes the conjugate of $H$ by $g \in G$. If $T$ is a subset of $G, H^{T}=\left\langle H^{t} \mid t \in T\right\rangle$. If $H$ and $K$ are subgroups of $G$, $H K$ is the set $\{h k \mid h \in H, k \in K\}$ and $[H, K]=\langle[h, k] \mid h \in H, k \in K\rangle$, where $[h, k]=h^{-1} k^{-1} h k$. Define $\left[H,{ }_{n} K\right]$ inductively by $\left[H,{ }_{0} K\right]=H$ and $\left[H,{ }_{i+1} K\right]=\left[\left[H,{ }_{i} K\right], K\right]$.

1.4. A class of groups is a collection of groups $\mathfrak{X}$ such that $1 \in \mathfrak{X}$ and whenever $G \in \mathfrak{X}$ and $G_{1}$ is isomorphic to $G$, then $G_{1} \in \mathfrak{X}$. We let

$$
\begin{aligned}
\mathfrak{F}= & \text { the class of finite groups, } \\
\mathfrak{G}= & \text { the class of finitely generated groups, } \\
\mathfrak{F}^{s n}= & \text { the class of groups, all of whose subnormal subgroups } \\
& \text { are finitely generated, } \\
\hat{\mathfrak{M}}\left(\hat{\mathfrak{M}}_{s}\right)= & \text { the class of groups satisfying the maximal condition for } \\
& \text { subgroups (subnormal subgroups). }
\end{aligned}
$$

If $\mathfrak{X}$ is a class of groups, we let

$s n \mathfrak{X}=$ class of subnormal subgroups of $\mathfrak{X}$-groups,

$q \mathfrak{X}=$ class of quotients of $\mathfrak{X}$-groups,

$n_{0} \mathfrak{X}=$ class of products of finitely many normal $\mathfrak{X}$-subgroups.

If $\varphi \in\left\{s n, q, n_{0}, s_{0}\right\}$, we say that the class $\mathfrak{X}$ is $\varphi$-closed if $\varphi \mathfrak{X}=\mathfrak{X}$. If $Y \subseteq\left\{s n, q, n_{0}, s_{0}\right\}, \mathfrak{X}=Y \mathfrak{X}$ if $\mathfrak{X}$ is $\varphi$-closed for all $\varphi \in Y$. If $\mathfrak{X}$ and $\mathfrak{Y}$ are two classes of groups, $\mathfrak{X} \mathfrak{Y}$ denotes the class of $\mathfrak{X}$-by- $\mathfrak{Y}$ groups and $\mathfrak{X} \wedge \mathfrak{Y}$ denotes the intersection of $\mathfrak{X}$ and $\mathfrak{Y}$. 


\section{Some preliminaries.}

2.1. LEMMA [1, LeMma 3.21]. Let $H$ and $K$ be subgroups of a group, let $N \triangleleft K$ and suppose $K / N$ can be generated by $n$ elements. Then, for any $t>0, H^{K}=L^{N}\left[H,{ }_{t} K\right]$ where $L$ is generated by at most $1+n+n^{2}+\cdots+$ $n^{t-1}$ conjugates of $H$ by elements of $K$.

2.2. LEMMA [2, LEMMA 2.4]. Let $\mathfrak{X}=\left\{s n, n_{0}\right\} \mathfrak{X}$. If $H \triangleleft \triangleleft G, K \triangleleft \triangleleft G$, $J=\langle H, K\rangle$ and $J=H K$, then $J \triangleleft \triangleleft G$; also $J \in \mathfrak{X}$ if $H \in \mathfrak{X}$ and $K \in \mathfrak{X}$.

We will need the main results of [3].

2.3. Definition [3, p. 423]. The class $\mathfrak{X}$ is locally coalescent if whenever $H$ and $K$ are subnormal $\mathfrak{X}$-subgroups of $G$, then every finitely generated subgroup $F$ of $J=\langle H, K\rangle$ is contained in some subnormal $\mathfrak{X}$-subgroup $X$ of $G$ such that $F \subseteq X \subseteq J$.

2.4. TheOrem [3, Theorems A AND B]. If $\mathfrak{X}$ is a class of groups such that $\mathfrak{X}=\left\{s n, n_{0}\right\} \mathfrak{X}$, then $\mathfrak{X}$ is locally coalescent. If $\mathfrak{X}$ is a locally coalescent class, then $\mathfrak{X} \wedge \mathfrak{G}$ is a subnormal coalition class.

2.5. DEFINITION. If $\mathfrak{X}$ is a class of groups, then

$$
\left.\theta_{\mathfrak{X}}(G)=\langle H| H \triangleleft \triangleleft G \text { and } H \in \mathfrak{X}\right\rangle .
$$

2.6. The following are immediate consequences of Definition 2.5:

(i) $\theta_{\mathfrak{X}}(G)$ is a characteristic subgroup of $G$.

(ii) If $K$ is a finitely generated subgroup of $\theta_{\mathfrak{X}}(G)$, there exist finitely many subnormal $\mathfrak{X}$-subgroups $H_{1}, H_{2}, \cdots, H_{n}$ of $G$ such that $K \subseteq\left\langle H_{1}, H_{2}, \cdots, H_{n}\right\rangle$.

(iii) If $\mathfrak{X}=\operatorname{sn} \mathfrak{X}$ is a subnormal coalition class and $K \triangleleft \triangleleft G$, then $\theta_{\mathfrak{X}}(K)=$ $\theta_{\mathfrak{X}}(G) \cap K$.

2.7. LEMMA [3, p. 424]. Let $\mathfrak{X}$ be a locally coalescent class. If $\theta_{\mathfrak{X}}(G)=G$, then every finitely generated subgroup of $G$ is contained in some subnormal $\mathfrak{X}$-subgroup of $G$.

2.8. Lemma. Let $\mathfrak{X}=s_{0} \mathfrak{X}$ and let $\mathfrak{Y}=\{s n, q\} \mathfrak{Y} \subseteq \mathfrak{G}$. If $G \in \mathfrak{X} \mathfrak{Y}$, then $\theta_{\mathfrak{X}}(G) \in \mathfrak{X}$ and $G / \theta_{\mathfrak{X}}(G) \in \mathfrak{Y}$.

Proof. Let $G \in \mathfrak{X Y Y}$ and let $N \triangleleft G$ such that $N \in \mathfrak{X}$ and $G / N \in \mathfrak{Y}$. Since $N \subseteq \theta_{\mathfrak{X}}(G), \theta_{\mathfrak{X}}(G) / N \in \mathfrak{Y} \subseteq \mathfrak{G}$. It follows from 2.6 (ii) that $\theta_{\mathfrak{X}}(G) \in$ $s_{0} \mathfrak{X}=\mathfrak{X}$. Since $q \mathfrak{Y}=\mathfrak{Y}$, we have $G / \theta_{\dot{\mathfrak{X}}}(G) \in \mathfrak{Y}$.

2.9. LEMMA. If $\mathfrak{X}=\left\{s n, n_{0}\right\} \mathfrak{X}$ and $\mathfrak{Y}=\left\{s n, q, n_{0}\right\} \mathfrak{Y}$ are classes such that whenever $A \in \mathfrak{X} \mathfrak{Y}, \theta_{\mathfrak{X}}(A) \in \mathfrak{X}$, then the class $\mathfrak{X} \mathfrak{Y}$ is locally coalescent.

Proof. By 2.4 , it suffices to show that $\mathfrak{X} Y=\left\{s n, n_{0}\right\} \mathfrak{X}$. 
Let $H$ and $K$ be normal $\mathfrak{X}$-subgroups of $J=\langle H, K\rangle$ and let $F_{H}=\theta_{\mathfrak{X}}(H)$ and $F_{K}=\theta_{\mathfrak{X}}(K)$. By hypothesis, $F_{H}, F_{K} \in \mathfrak{X}$ and $H / F_{H}, K / F_{K} \in q \mathfrak{Y}=\mathfrak{Y}$. It follows that $F_{H}$ and $F_{K}$ are normal $\mathfrak{X}$-subgroups of $J$ and $F_{H} F_{K} \in \mathfrak{X}=$ $n_{0} \mathfrak{X}$. Hence, $J / F_{H} F_{K} \in\left\{q, n_{0}\right\} \mathfrak{Y}=\mathfrak{Y}$ and $\mathfrak{X} \mathfrak{Y}=n_{0} \mathfrak{X} \mathfrak{Y}$.

If $H \triangleleft G \in \mathfrak{X Y}$, then $K=\theta_{\mathfrak{X}}(G) \in \mathfrak{X}$ and $G / K \in \mathfrak{Y}$. Hence, $H K / K \triangleleft G / K$ and $H K \mid K \in \mathfrak{Y}=s n \mathfrak{Y}$. Also, $H \cap K \in \mathfrak{X}=s n \mathfrak{X}$. It follows that $H \in \mathfrak{X} \mathfrak{Y}$ and $\mathfrak{X} \mathfrak{Y}=s n \mathfrak{X}$.

\section{Subnormal coalition classes.}

3.1. LEMMA. Let $\mathfrak{X}$ be a subnormal coalition class and let $\mathfrak{Y}=\left\{s n, q, n_{0}\right\} \mathfrak{Y}$ be $a$ class of groups such that whenever $A \in \mathfrak{X Y}, \theta_{\mathfrak{X}}(A) \in \mathfrak{X}$. Let $H$ and $K$ be subnormal $\mathfrak{X}$-subgroups of $G$ such that $\langle H, K\rangle=H K$. If $N \triangleleft H$ such that $N \in \mathfrak{X}, H / N \in \mathfrak{Y}$, and $\langle N, K\rangle$ is a subnormal $\mathfrak{X Y}$-subgroup of $G$, then $\langle H, K\rangle$ is a subnormal $\mathfrak{X Y}$-subgroup of $G$.

Proof. It follows from 2.2 that $H K$ is subnormal in $G$.

Let $M=\langle N, K\rangle$ and let

$$
M=M_{m} \triangleleft M_{m-1} \triangleleft \cdots \triangleleft M_{1} \triangleleft M_{0}=H K
$$

be the standard series for $M$ in $H K$. Suppose $M_{i+1} \in \mathfrak{X Y}$. Then $\theta_{\mathfrak{X}}\left(M_{i+1}\right)=$ $\bar{M} \in \mathfrak{X}$ and $\bar{M} \triangleleft M_{i}$. Now,

and

$$
M_{i}^{\prime}=M_{i} \cap H K=\left(M_{i} \cap H\right) M_{i+1}
$$

$$
M_{i} / \bar{M}=\left(\left(M_{i} \cap H\right) \bar{M} / \bar{M}\right)\left(M_{i+1} / \bar{M}\right) .
$$

Since $\left(M_{i} \cap H\right) \bar{M} / \bar{M}$ and $M_{i+1} / \bar{M}$ are subnormal $\mathfrak{Y}=\left\{s n, n_{0}\right\} \mathfrak{Y}$-subgroups of $M_{i} \mid \bar{M}$, it follows from 2.2 that $M_{i} \mid \bar{M} \in \mathfrak{Y}$. Hence, $M_{i} \in \mathfrak{X Y}$ and $H K \in \mathfrak{X Y}$.

3.2. Lemma. Let $\mathfrak{X}$ be a subnormal coalition class and let $\mathfrak{Y}=$ $\left\{s n, q, n_{0}\right\} \mathfrak{Y}$ be a class of groups such that whenever $A \in \mathfrak{X Y}, \theta_{\mathfrak{X}}(A) \in \mathfrak{X}$. If $H$ is a subnormal $\mathfrak{X}(\mathfrak{Y} \wedge(\mathfrak{5})$-subgroup and $K$ is a subnormal $\mathfrak{X}$-subgroup of $G$, then $\langle H, K\rangle$ is a subnormal $\mathfrak{X}(\mathfrak{Y} \wedge(\mathfrak{b})$-subgroup of $G$.

Proof. Let $N=\theta_{\mathfrak{X}}(H), J=\langle H, K\rangle$, and $\bar{J}=\left\langle K^{H}, N\right\rangle$. By hypothesis, $N \in \mathfrak{X}$ and $H / N \in q(\mathfrak{Y} \wedge(\mathfrak{5})=\mathfrak{Y} \wedge(5$. If $t=s(G, H)$, it follows from 2.1 that

$$
\bar{J}=\left\langle L^{N}\left[K,{ }_{t} H\right], N\right\rangle=\langle L, N\rangle\left\langle\left[K,{ }_{t} H\right], N\right\rangle,
$$

where $L$ is the join of a finite number of conjugates of $K$. Since $\mathfrak{X}$ is a subnormal coalition class, $M=\langle L, N\rangle$ is a subnormal $\mathfrak{X}$-subgroup of $G$. Since $N \subseteq\left\langle\left[K,{ }_{t} H\right], N\right\rangle \triangleleft H$ and $\mathfrak{Y}=q \mathfrak{Y},\left\langle\left[K,{ }_{t} H\right], N\right\rangle$ is a subnormal $\mathfrak{X Y}$ subgroup of $G$. Consequently, by $2.2, J \triangleleft \triangleleft G$ and $J=J H \triangleleft \triangleleft G$. An 
application of 3.1 shows that $\bar{J} \in \mathfrak{X Y}$. A second application of 3.1 shows that $J=J H \in \mathfrak{X} Y$. Since $\theta_{\mathfrak{X}}(J) \in \mathfrak{X}$ and $J / \theta_{\mathfrak{X}}(J) \in \mathfrak{F}, J \in \mathfrak{X}(\mathfrak{Y} \wedge(\mathfrak{F})$.

ProOF OF THEOREM A. Let $H$ and $K$ be subnormal $\mathfrak{X}(\mathfrak{Y} \wedge(\mathfrak{F})$ subgroups of $G$ and let $J=\langle H, K\rangle$. Let $N \triangleleft H$ such that $N \in \mathfrak{X}$ and $H / N \in$ $\mathfrak{Y} \wedge \mathfrak{F}$.

If $t=s(G, H)$, it follows from 2.1 that

$$
\left\langle K^{H}, N\right\rangle=\left\langle L^{N}\left[K,{ }_{t} H\right], N\right\rangle=\langle L, N\rangle\left\langle\left[K,{ }_{t} H\right], N\right\rangle,
$$

where $L$ is the join of a finite number of conjugates of $K$. By induction on $s(G, K)$ we conclude that $L$ is a subnormal $\mathfrak{X}(\mathfrak{V} \wedge(\mathfrak{5})$-subgroup of $G$. An application of 3.2 shows that $\langle L, N\rangle$ is a subnormal $\mathfrak{X}(\mathfrak{Y} \wedge(\mathfrak{G})$-subgroup of $G$. Also, $\left\langle\left[K,{ }_{t} H\right], N\right\rangle \in \mathfrak{X Y Y}$. It follows from 3.1 that $\left\langle K^{H}, N\right\rangle \in \mathfrak{X Y}$ and from 2.2 that $\left\langle K^{H}, N\right\rangle \triangleleft \triangleleft G$. Since $J=\left\langle K^{H}, N\right\rangle H$, it follows from 3.1 that $J \in \mathfrak{X Y}$ and from 2.2 that $J \triangleleft \triangleleft G$. Since $\theta_{\mathfrak{X}}(J) \in \mathfrak{X}$ and $J / \theta_{\mathfrak{X}}(J) \in \mathfrak{F}$, $J \in \mathfrak{X}(\mathfrak{Y} \wedge(\mathfrak{5})$.

\section{4. $s_{0}$-closed classes.}

4.1. LEMMA. Let $\mathfrak{X}=s_{0} \mathfrak{X}$ and $\mathfrak{Y}=\{s n, q\} \mathfrak{Y} \subseteq \mathfrak{G}$ be two classes of groups. If $H=\left\langle H_{1}, H_{2}, \cdots, H_{n}\right\rangle$, where $H_{i}$ is a subnormal $\mathfrak{X}$-subgroup of $G$, and $K$ is a subnormal $\mathfrak{X Y}$-subgroup of $G$, then $\langle H, K\rangle$ is an $\mathfrak{X Y}$-subgroup of $G$.

Proof. Since $K \in \mathfrak{X} \mathfrak{Y}$, there exists $N \triangleleft K$ such that $N \in \mathfrak{X}$ and $K / N \in \mathfrak{Y}$. If $t=s(G, K)$, an application of 2.1 shows that $\left\langle H^{K}, N\right\rangle=\left\langle L,\left[H,{ }_{t} K\right], N\right\rangle$, where $L$ is the join of a finite number of conjugates of $H$. Since $\left[H,{ }_{t} K\right] \triangleleft K$, $\left[H,{ }_{t} K\right] N / N \in \mathfrak{V}=s n \mathfrak{Y} \subseteq \mathfrak{G}$ and there exist finitely many elements $x_{1}, x_{2}, \cdots, x_{l} \in\left[H,{ }_{t} K\right]$ such that

$$
\left\langle\left[H,{ }_{t} K\right], N\right\rangle=\left\langle x_{1}, x_{2}, \cdots, x_{l}, N\right\rangle .
$$

Since $\left[H,{ }_{t} K\right] \subseteq H^{K}$, there exist finitely many elements $k_{1}, k_{2}, \cdots, k_{m} \in K$ such that

$$
\left\langle x_{1}, x_{2}, \cdots, x_{l}\right\rangle \subseteq\left\langle H^{k_{1}}, H^{k_{2}}, \cdots, H^{k_{m}}\right\rangle
$$

Consequently,

$$
\left\langle H^{K}, N\right\rangle=\left\langle L, H^{k_{1}}, H^{k_{2}}, \cdots, H^{k_{m}}, N\right\rangle \in \mathfrak{X}=s_{0} \mathfrak{X} .
$$

But then $\langle H, K\rangle /\left\langle H^{K}, N\right\rangle \in q \mathfrak{Y}=\mathfrak{Y}$ and $\langle H, K\rangle \in \mathfrak{X} \mathfrak{Y}$.

ProOF OF THEOREM C. Let $G=\left\langle H_{1}, H_{2}, \cdots, H_{n}\right\rangle$, where $H_{i}$ is a subnormal $\mathfrak{X}$-subgroup of $G, 1 \leqq i \leqq n$. Let $F_{i}=\theta_{\mathfrak{X}}\left(H_{i}\right)$. By $2.8, F_{i} \in \mathfrak{X}$ and $H_{i} \mid F_{i} \in \mathfrak{Y} \subseteq \mathfrak{F}$. Let $T_{i}$ be a finite subset of $H_{i}$ such that $H_{i}=\left\langle F_{i}, T_{i}\right\rangle$ and let $T=\bigcup_{i=1}^{n} T_{i}$.

Since $\mathfrak{X}$ and $\mathfrak{Y}$ satisfy the hypothesis of 2.9 , the class $\mathfrak{X} \mathfrak{Y}$ is locally 
coalescent. There exists by 2.7 a subnormal $\mathfrak{X}$-subgroup $K$ of $G$ such that $\langle T\rangle \subseteq K$. An application of 4.1 shows that $\left\langle F_{1}, F_{2}, \cdots, F_{n}, K\right\rangle=G \in$ $\mathfrak{X Y}$.

4.2. Lemma. Suppose $H \triangleleft G,|G: H|=n<\infty$, and $K \subseteq H$. If $A=\{1=a(1)$, $a(2), \cdots, a(n)\}$ is a right transversal for $H$ in $G$ such that $G=\langle K, A\rangle$, then there exists a finite subset $L$ of $H$ such that $H=\left\langle K^{a}, L \mid a^{-1} \in A\right\rangle$.

Proof. Let $a, b \in A$ and $k \in K$. Since $K \subseteq H \triangleleft G$, we see that $a k b^{-1} \in H$ if and only if $a=b$. For all $a(i), a(j) \in A, 1 \leqq i, j \leqq n$, we define $a(i, j) \in A$ uniquely by the equation $a(i) a(j) a(i, j)^{-1} \in H$.

Let $\bar{H}$ be defined by

$$
\bar{H}=\left\langle K^{a}, a(i) a(j) a(i, j)^{-1} \mid a^{-1} \in A, 1 \leqq i, j \leqq n\right\rangle .
$$

Since $H \triangleleft G, \bar{H} \subseteq H$. If $g \in H$, then $g=g_{1}^{\varepsilon_{1}} g_{2}^{\varepsilon_{2}} \cdots g_{m}^{\varepsilon_{m}}$ for some elements $g_{i} \in K \cup A$ and $\varepsilon_{i}= \pm 1,1 \leqq i \leqq m$. Set $a\left(i_{0}\right)=1$. There exists a unique element $a\left(i_{1}\right) \in A$ such that $a\left(i_{0}\right) g_{1}^{\varepsilon_{1}} a\left(i_{1}\right)^{-1} \in H$. It is easily verified that $\left(a\left(i_{0}\right) g_{1}^{\varepsilon_{1}} a\left(i_{1}\right)^{-1}\right)^{\varepsilon_{1}}$ is a displayed generator of $\bar{H}$. Suppose that for all $j$, $1 \leqq j<l \leqq m$, we have chosen $a\left(i_{j}\right)$ such that $\left(a\left(i_{j-1}\right) g_{j}^{\varepsilon_{j}} a\left(i_{j}\right)^{-1}\right)^{\varepsilon_{j}}$ is a generator of $\bar{H}$. We then choose $a\left(i_{l}\right) \in A$ as the unique element satisfying the equation $a\left(i_{l-1}\right) g_{l}^{\varepsilon_{l}} a\left(i_{l}\right)^{-1} \in H$. Again, $\left(a\left(i_{l-1}\right) g_{l}^{\varepsilon_{l}} a\left(i_{l}\right)^{-1}\right)^{\varepsilon_{l}}$ is a generator of $\bar{H}$. But then

$$
g=a\left(i_{0}\right) g_{1}^{\varepsilon_{1}^{1}} a\left(i_{1}\right)^{-1} a\left(i_{1}\right) g_{2}^{\varepsilon_{2}^{2}} a\left(i_{2}\right)^{-1} \cdots a\left(i_{m-1}\right) g_{m}^{\varepsilon} a\left(i_{m}\right)^{-1} a\left(i_{m}\right),
$$

where $a\left(i_{l-1}\right) g_{l}^{\varepsilon_{l}} a\left(i_{l}\right)^{-1} \in \bar{H}, 1 \leqq l \leqq m$. Since $\bar{H} \subseteq H, a\left(i_{m}\right)=1$. Hence, $g \in \bar{H}$ and $H=\bar{H}$. The lemma follows if we set $L=\left\{a(i) a(j) a(i, j)^{-1} \mid 1 \leqq\right.$ $i, j \leqq n\}$.

Proof of Theorem D. Let $G=\left\langle H_{1}, H_{2}, \cdots, H_{n}\right\rangle$ where $H_{i}$ is a subnormal $\mathfrak{X} \mathfrak{F}$-subgroup of $G, 1 \leqq i \leqq n$. Let $F_{i}=\theta_{\mathfrak{X}}\left(H_{i}\right)$. By $2.8, F_{i} \in \mathfrak{X}$ and $H_{i} \mid F_{i} \in \mathfrak{F}$. Since $F_{i} \subseteq \theta_{\mathfrak{X}}(G)$, it follows that $H_{i} \theta_{\mathfrak{X}}(G) / \theta_{\mathfrak{X}}(G)$ is a finite subnormal subgroup of $G / \theta_{\mathfrak{X}}(G)$. It is a consequence of 2.4 that $\mathfrak{F}$ is a subnormal coalition class. Consequently, $G / \theta_{\mathfrak{X}}(G) \in \mathfrak{F}$.

Let $A$ and $A_{i}, 1 \leqq i \leqq n$, be right transversals for $\theta_{\mathfrak{x}}(G)$ in $G$ and $F_{i}$ in $H_{i}$ respectively such that $1 \in A$. Since $G=\left\langle H_{1}, H_{2}, \cdots, H_{n}\right\rangle$,

$$
G=\left\langle F_{1}, F_{2}, \cdots, F_{n}, A_{1}, A_{2}, \cdots, A_{n}\right\rangle .
$$

There exists a finite subset $U$ of $\theta_{\mathfrak{X}}(G)$ such that $G=\left\langle F_{1}, F_{2}, \cdots, F_{n}\right.$, $U, A\rangle$. By 2.6(ii), there exist a finite number of subnormal $\mathfrak{X}$-subgroups $L_{1}, L_{2}, \cdots, L_{l}$ of $G$ such that $\langle U\rangle \subseteq\left\langle L_{1}, L_{2}, \cdots, L_{l}\right\rangle$. If we let $K=\left\langle F_{i}, L_{j} \mid 1 \leqq i \leqq n, 1 \leqq j \leqq l\right\rangle$, an application of 4.2 shows the existence of a finite subset $V$ of $\theta_{\mathfrak{X}}(G)$ such that

$$
\theta_{\mathfrak{X}}(G)=\left\langle K^{a}, V \mid \sigma^{-1} \in A\right\rangle .
$$


By 2.6, there exist a finite number of subnormal $\mathfrak{X}$-subgroups $M_{1}, M_{2}, \cdots$, $M_{m}$ of $G$ such that $\langle V\rangle \subseteq\left\langle M_{1}, M_{2}, \cdots, M_{m}\right\rangle$. Hence, $\theta_{\mathfrak{X}}(G)$ is the join of a finite number of subnormal $\mathfrak{X}$-subgroups and $\theta_{\mathfrak{X}}(G) \in \mathfrak{X}=s_{0} \mathfrak{X}$.

\section{REFERENCES}

1. D. J. S. Robinson, Infinite soluble and nilpotent groups, Queen Mary College Math. Notes, Queen Mary College, London, 1967. MR 42 \#4635.

2. Derek S. Robinson, Joins of subnormal subgroups, Illinois J. Math. 9 (1965), 144-168. MR 30 \#1187.

3. J. E. Roseblade and S. E. Stonehewer, Subjunctive and locally coalescent classes of groups, J. Algebra 8 (1968), 423-435. MR 36 \#5208.

4. S. E. Stonehewer, The join of finitely many subnormal subgroups, Bull. London Math. Soc. 2 (1970), 77-82. MR 41 \#1877.

Department of Mathematics, Michigan State University, East lansing, MiChIGAN 48823

Current address: Aetna Life and Casualty, Hartford, Connecticut 06105 